Relations industrielles

Industrial Relations

\title{
Collection "Technologie et travail". Montréal, Institut national de productivité, 1983, 8 textes de 43 pp. à 202 pp., ISBN \\ 2-7081-0541-8
}

\section{Jean Nollet}

Volume 39, numéro 2, 1984

URI : https://id.erudit.org/iderudit/050043ar

DOI : https://doi.org/10.7202/050043ar

Aller au sommaire du numéro

Éditeur(s)

Département des relations industrielles de l'Université Laval

ISSN

0034-379X (imprimé)

1703-8138 (numérique)

Découvrir la revue

Citer ce compte rendu

Nollet, J. (1984). Compte rendu de [Collection "Technologie et travail". Montréal, Institut national de productivité, 1983, 8 textes de 43 pp. à 202 pp., ISBN 2-7081-0541-8]. Relations industrielles / Industrial Relations, 39(2), 400-402. https://doi.org/10.7202/050043ar

Tous droits réservés @ C Département des relations industrielles de l'Universite Laval, 1984
Ce document est protégé par la loi sur le droit d'auteur. L'utilisation des services d’Érudit (y compris la reproduction) est assujettie à sa politique d'utilisation que vous pouvez consulter en ligne.

https://apropos.erudit.org/fr/usagers/politique-dutilisation/ 
Les impacts de l'informatisation des méthodes de gestion et de production dans les petites et moyennes imprimeries générales du Québec, 70 pp., 1983, ISBN 2-551-05818-X.

La collection Technologie et Travail comprend huit textes abordant différentes facettes de la technologie et les effets qu'elle entraîne. Les quatre premiers comportent chacun moins de cinquante pages et abordent successivement les microprocesseurs, la bureautique, la robotique, puis la conception et la fabrication assistées par ordinateur (CFAO). Les trois suivants apportent une vision plus globale de l'impact des nouvelles technologies sur les plans économique, social, puis politique. Le dernier texte clôture cette collection par l'étude d'une situation pratique d'application de nouvelles technologies dans une industrie québécoise et des conséquences qu'elle a entraînées.

Les textes sont écrits par des auteurs distincts, ce qui rend parfois les styles passablement différents d'un ouvrage à l'autre. Cependant, tous sont rendus dans un style clair et contiennent de nombreuses sections, ce qui en facilite la lecture. Ces 600 pages constituent un tour d'horizon intéressant et ne visant naturellement pas à présenter une étude exhaustive de la situation. De même, l'objectif visé n'est pas de repousser les frontières du savoir, mais bien de décrire et de diffuser les principales facettes connues des domaines abordés.

Introduction à la technologie des microprocesseurs donne une bonne vue d'ensemble de la technologie microélectronique. Ce texte fournit une foule d'exemples concrets et permet de placer les sept autres textes dans un contexte approprié.

L'avant-propos de chacun des trois documents suivants spécifie clairement que ce sont là des «documents sommaires donnant une vue d'ensemble ... et que chacune de ces études ... est destinée à des gens initiés, mais non spécialisés». Introduction à la bureautique décrit ce qu'est la bureautique et fournit certains résultats obtenus, suite à l'utilisation de cette technologie. Le glossaire reproduit de l'une des références est clair et utile.

Introduction a la robotique est un texte extrêmement pratique et sera particulièrement apprécié par ceux qui désirent un aperçu concret de ce qu'est la robotique, mais aussi par les gestionnaires considérant implanter des robots. Ce document comporte une vingtaine de figures, pour la plupart des robots à l'oeuvre. L'exemple d'application et les liens de la robotique avec la productivité sont deux autres contributions que le lecteur appréciera. Introduction à la CFAO se caractérise également par une approche pratique, facilitant la compréhension des possibilités de la CFAO. L'effort de vulgarisation est particulièrement appréciable dans ce cas-ci, car il s'agit sans doute là d'une notion plus difficile à cerner et à présenter que la bureautique et la robotique.

$\mathrm{Y}$ a-t-il des traits communs quant à l'introduction des nouvelles technologies? Certes oui. C'est ce que L'introduction de technologies nouvelles dans l'entreprise: facteurs et conséquences met en relief. Ce document présente une discussion intéressante, plus poussée sur le plan académique que les quatre textes précédents. Par contre, il est suffisamment clair pour que tout lecteur intéressé au processus d'introduction de nouvelles technologies y trouve son compte. L'effort d'intégration de cette étude d'une cinquantaine de pages - à double interligne - est remarquable. Comme beaucoup d'ouvrages servant de base à ce texte comportent des études ou des observations tirées de la pratique, les généralisations faites dans cette études pourraient être d'autant plus utiles aux gestionnaires et aux hauts fonctionnaires.

Nouvelles technologies et caractéristiques du travail saura certes intéresser les lecteurs de Relations industrielles. En s'appuyant sur une bibliographie imposante, les auteurs soulèvent une foule de réflexions dans l'esprit du lecteur. C'est un texte dense, mais bien entrecoupé d'exemples concrets, simples à saisir et illustrant bien les éléments 
conceptuels apportés. Cette approche facilite les passages d'un concept au suivant: le lecteur constatera que beaucoup d'idées intéressantes y sont émises, tant pour le travail dans le secteur secondaire que dans le secteur tertiaire.

Le texte suivant, intitulé Technologie et emploi: un inventaire de stratégies nationales, décrit pour dix pays industrialisés - huit d'Europe, le Japon et les États-Unis - les stratégies et politiques nationales employées face à l'introduction de nouvelles technologies et aux conséquences de ces dernières sur l'emploi. Ce texte permet en même temps de visualiser le contexte économique et social propre à chacun de ces pays.

Finalement, Les impacts de l'informatisation dans les imprimeries du Québec rapporte les résultats d'une recherche effectuée au sein d'une industrie typiquement composée de PME. Encore une fois, cette étude suppose concrètement l'hypothèse selon laquelle il y a de nombreux points communs dans une même industrie, quant à la gestion de l'introduction de technologies. Cependant, les différences sont tout aussi remarquables et ne peuvent être négligées.

Globalement, ces huit textes fournissent une toile de fond intéressante quant aux caractéristiques des nouvelles technologies et à leur impact, tant au niveau de l'entreprise qu'au niveau national. Ces documents descriptifs sont orientés vers la pratique et sont facilement compréhensibles. L'un des avantages principaux réside dans le fait qu'il soit possible de lire n'importe lequel sans nécessairement avoir à lire tous les autres. Nul doute que ces textes peuvent sûrement aider et intéresser tout lecteur concerné par le virage technologique et par ses conséquences.

Jean NOLLET

Ecole des Hautes Etudes

Commerciales de Montréal
Le droit du travail en pratique, par Yves Delamotte, Paris, Les Éditions d'organisation, 1983, 319 pp., ISBN 2-7081-0541-8

Comme il fallait s'y attendre avec l'avènement d'un gouvernement socialiste au pouvoir, la législation du travail en France a connu des transformations profondes en 1982. Il ne s'agit pas seulement d'un rajeunissement mais d'une réforme marquant un tournant comme celles qui avaient été effectuées en $1864,1884,1936,1946$ et 1950. Un tiers du Code du travail a été abrogé et remplacé par des dispositions législatives nouvelles.

L'ouvrage du professeur Delamotte tient compte de ces développements et cherche à en dégager la portée. Il n'a pas été conçu à l'intention des étudiants en droit ni des juristes, mais plutôt pour ceux qui veulent acquérir des connaissances en droit du travail et particulièrement pour ceux qui, dans la pratique de leur vie professionnelle, ont besoin de connaître les règles qui régissent la situation des salariés et les rapports collectifs du travail, d'où le titre Le droit du travail en pratique.

Après une courte introduction historique, l'ouvrage est constitué de vingt-quatre chapitres classés en quatre parties. Suivent plusieurs annexes, des indications bibliographiques et enfin un index onomastique.

La première partie présente les organismes administratifs en matière de travail et d'emploi ainsi que les sources du droit. La seconde est consacrée à l'accès et à la perte de l'emploi ainsi qu'aux aléas de la vie professionnelle. Elle traite des différentes sortes de contrat de travail: à durée déterminée, travail temporaire, travail à temps partiel, de la résiliation du contrat de travail, du licenciement, de la cessation du contrat et enfin des prérogatives de la direction.

La troisième partie aborde le salaire et les conditions de travail: le salaire; participation et actionnariat; durée de travail et aménagement du taux de travail; jours fériés et congés; hygiène et sécurité; comité d'hygiène, de sécurité et des conditions de travail ainsi 